\title{
Psychopathology and adaptive functioning of parents of adolescents with anorexia nervosa
}

\author{
FELIPE AlCKMin-CaRVAlHO 1,2 \\ https://orcid.org/0000-0002-0203-2650 \\ Juliana Bergamo Vega1 \\ https://orcid.org/0000-0001-9520-9760
}

Alicia Weisz Cobelo ${ }^{1}$

https://orcid.org/0000-0001-8232-5834

Vanessa Dentzien Pinzon ${ }^{1}$

https://orcid.org/0000-0002-8543-4662

1 Eating Disorders Outpatient and Inpatient Program, Child and Adolescent Psychiatry Division (PROTAD/SEPIA), Department of Psychiatry, Institute of Psychiatry, University of São Paulo (USP), São Paulo, SP, Brazil.

2 Clinical Psychology Program, Institute of Psychology, University of São Paulo (USP), São Paulo, SP, Brazil.

Work conducted at the Eating Disorders Outpatient and Inpatient Program, Child and Adolescent Psychiatry Division (PROTAD/SEPIA), Department of PSychiatry, Institute of Psychiatry, University of Sao Paulo (USP), São Paulo, SP, Brazil.

Received: 5/9/2019- Accepted: 6/25/2019

DOl: $10.1590 / 0101-60830000000213$

Alckmin-Carvalho F et al. / Arch Clin Psychiatry. 2019;46(5):141

\section{Dear Editor,}

Literature indicates that parents' participation in the treatment of their children with Anorexia Nervosa (AN) is associated with better prognosis ${ }^{1}$. In family-based treatment ${ }^{2}$, the most tested form of psychotherapy for AN in childhood and adolescence, parents are protagonists in the behavioral management for weight recovery and control of symptoms ${ }^{3}$. However, psychopathologies and adaptive difficulties of these parents may impair adherence to the therapies proposed by the interdisciplinary team ${ }^{4,5}$.

The aim of this letter is to present an evaluation of the adaptive functioning and psychopathology of parents of adolescents with AN in treatment at the Eating Disorders Outpatient and Inpatient Program, Child and Adolescent Psychiatry Division (PROTAD/ SEPIA), Institute of Psychiatry, University of Sao Paulo (USP), São Paulo, SP, Brazil.

This is a cross-sectional study involving 43 parents (mean age $=44.5 ; \mathrm{SD}=7.0$ ) of 33 children and adolescents (mean age $=14.8$; $\mathrm{SD}=0.9$ ), diagnosed with AN by DAWBA (DSM-IV-TR). For the evaluation of the adaptive functioning and psychopathology, the Brazilian version of the Adult Self-Report (ASR) was used. The ASR is one of the self-reported instruments of the Achenbach System of Empirically Based Assessment (ASEBA) ${ }^{6}$, used worldwide to evaluate psychopathologies in adults, based on the DSM-IV-TR diagnostic criteria, with evidence of validity for the Brazilian population ${ }^{7}$. The instrument was applied to all parents of patients with AN admitted at PROTAD between 2016 and 2018, both in the inpatient and outpatient settings.

The Research Ethics Committee for studies involving human subjects of the HCFMUSP approved the study (protocol number $0800 / 08$ ). The sample consisted mainly of mothers ( $n=34,79.0 \%)$. Among the participants evaluated, the majority reported being white $(\mathrm{n}=25,58.1 \%)$ and with higher education $(\mathrm{n}=34,83.7 \%)$. The results indicated that $10(23.5 \%)$ parents presented total adaptive functioning at clinical range, and the most impaired domains were marital $(n=17,44.7 \%)$ and work $(n=9,20.9 \%)$. In terms of psychopathology and cognitive/behavioral problems, 19 parents (44.2\%) presented the total scores at clinical range. The most prevalent mental health problems were: anxiety $(\mathrm{n}=21,48.8 \%)$, depression $(\mathrm{n}=15,34.9 \%)$; aggressive behavior $(n=14,32.5 \%)$; isolation $(n=13 ; 30.2 \%)$; somatic complaints $(n=13,30.2 \%)$; Attention-Deficit Hyperactivity Disorder $(n=9,20.9 \%)$ and antisocial personality $(n=9,20.9 \%)$. We found no statistically significant difference between the proportion of fathers and mothers at clinical range using $\mathrm{Z}$-Test.
The results indicated high prevalence of psychopathologies and cognitive/behavioral problems among parents of children and adolescents with $\mathrm{AN}$, at the beginning of treatment. These findings are in agreement with previous studies, which reported a high level of hostility, depression and anxiety in this population ${ }^{4,5}$.

These pre-existing difficulties can be aggravated by a long and stressful treatment. Therefore, the findings suggest the importance of providing psychotherapeutic and/or psychiatric care for the parents at the unit where their children are assisted, and referral to other mental health services, when necessary. It tends to optimize adherence to treatment of their children and decrease the risk of relapse. We emphasize the relevance of further research on mental health of parents of children and adolescents with AN, as well as the need to assess the impact of parental psychopathologies on the severity of cases of AN and on the effectiveness of the treatment.

\section{Disclosure}

The authors declare that there are no conflicts of interest.

\section{References}

1. Alckmin-Carvalho F, Bergamo JV, Cobelo AW, Fabbri A, Pinzon VD, Melo MHDS. Evidence-based psychotherapy for treatment of anorexia nervosa in children and adolescents: systematic review. Arch Clin Psychiatry. 2018;45(2):41-8.

2. Lock J, Le Grange D. Treatment manual for anorexia nervosa: a familybased approach. New York: Guilford Publications; 2015.

3. National Institute for Health and Clinical Excellence. Eating disorders: recognition and treatment NICE guideline NG69. 2017. Available from: https://www.nice.org.uk/guidance/ng69/chapter/Recommendations. Accessed on: Nov 08, 2018.

4. Ravi S, Forsberg S, Fitzpatrick K, Lock J. Is there a relationship between parental self-reported psychopathology and symptom severity in adolescents with anorexia nervosa? Eat Disord. 2008;17(1):63-71.

5. Rienecke RD, Richmond, RL. Psychopathology and expressed emotion in parents of patients with eating disorders: relation to patient symptom severity. Eat Disord. 2017;25(4):318-29.

6. Achenbach TM, Rescorla LA. Manual for the ASEBA Adult Forms \& Profiles [Manual]. Burlington, VT: University of Vermont, Research Center for Children, Youths, and Families; 2003.

7. Lucena-Santos P, Moraes JFD, Oliveira MS. Análise da estrutura fatorial das escalas sindrômicas do ASR (Adult Self-Report). Interam J Psychol. 2014;48(2):237-49. 